\title{
Producción baby leaf de berro de tierra cultivado en diferentes ambientes y marcos de plantación.
}

\author{
Jhone Souza Espíndola ${ }^{1}$, Rosana Fernandes Otto ${ }^{2}$, Gustavo Castilho Beruski ${ }^{3}$, Silvana Ohse ${ }^{2}$ \\ ${ }^{1}$ Centro Universitário Grupo Integrado, Campo Mourão, Paraná, Brasil. E-mail: jhone.souza@ grupointegrado.br \\ ${ }^{2}$ Universidade Estadual de Ponta Grossa, Campus Uvaranas, Ponta Grossa, Paraná, E-mail: rfotto@uepg.br, soshe@uepg.br \\ ${ }^{3}$ Universidade de São Paulo, Escola Superior de Agricultura Luiz de Queiroz, Piracicaba, São Paulo, Brasil. E-mail: \\ beruskigc@usp.br
}

Recebido: 30/03/2018; Aceito: 26/07/2018

\section{RESUMEN}

Baby leaf es un tipo de hortícola que llama la atención de los consumidores por el pequeño tamaño de las hojas y la posibilidad de ser comercializada con una mezcla de hojas para ensalada. El trabajo se realizó en Ponta Grossa- PR (Brasil) y tuvo como objetivo evaluar los ambientes de cultivo y la densidad plantas en la producción de baby leaf de berros de tierra. Los tratamientos fueron 03 ambientes de cultivo (TA - túnel agrotextil; TP - túnel plástico; ALAire Libre) y 03 marcos de plantación (E1- 15 x $10 \mathrm{~cm}$; E2- 15 x 7 cm; E3- 10 x 7 cm).Los ambientes TP y TA, de manera independiente, proporcionaron altura y número de hojas de plantas superiores al cultivo en AL, así como valores mayores de biomasas fresca (BF) y seca (BS) y del índice de área foliar (IAF).Los rendimientos totales de las plantas cultivadas bajo protección (TP y TA) fueron hasta 50\% superiores a los resultados encontrados en AL. Con relación a lo marco de plantación se encontró respuestas significativas para IAF, BF, BS y rendimiento total (RT). Las plantas se presentaran firmes y tiernas en todos los marcos de plantación evaluados, manteniendo la calidad comercial. La producción de baby leaf de berros de tierra fue viable bajo túneles cubiertos con agrotextil (TA) o plástico (TP) para la época primavera, utilizando el marco de plantación 10 x $7 \mathrm{~cm}$ (E3) entre plantas.

Palavras-chave: Barbarea verna, microclima, túnel bajo, cultivo protegido

\section{Yield of land cress baby leaf grown in different environments and plant densities}

\begin{abstract}
Baby leaf is a kind of vegetable that draws attention of consumers by the small size of leaves and for possibility to be marketed as a mix leaves of salad. The study was carried out in Ponta Grossa - PR (Brazil) and the aim was to assess different environmental conditions and plant densities for land cress baby leaf yield. The treatments were three environmental conditions (TA - nonwoven tunnel; TP - plastic tunnel; and AL - natural conditions) and three plant spacing (E1- 15x10 cm;E2 - 15x7 cm and E3 - 10x7 cm). The TP and TA, Independently, environments provided greater height plants and number of leaves compared to plants cultivated in AL conditions, even as greater fresh (BF) and weight (BS) masses of plant and neither leaf area index (IAF). The productivity (RT) of plants growing under protection conditions (TP and TA) were up 50\% higher than the amount of AL plants. For plant densities, there were significant differences to IAF, BF, BS and RT characteristics. The plants showed firm and tender with commercial quality in all spacing arrange studied. It was possible to product cress land baby leaf under row tunnels cover with plastic or nonwoven during the spring of Ponta Grossa - PR using 10x7 cm spacing between plants.
\end{abstract}

Key-words: Barbarea verna; microclimate; low tunnel; protect cultivation 


\section{Introducción}

A finales de los años 90 hubo aumento significativo en la producción y consumo de hortícolas mínimamente procesados (IV gama) en varios países. Factores como los cambios en el estilode vida, el creciente de mujeres en el mercado laboral y los hogares con 2-3 miembros favorecieron el consumo de este producto. Las hortícolas de hoja son las más consumidas, con crecimiento del tipo baby leaf. Todavía son productos delicados y muy susceptibles a procesos de manipulación (Nicola et al., 2006). Además, baby leaf no es un producto muy conocido en algunos países, como es el caso del mercado brasileño.

Las hortalizas tipo baby leaf son atractivas por el reducido tamaño, tierna consistencia y diferenciado sabor Baby leaf se produce con el cultivo de especies vegetales cultivadas, tales como berro, lechuga, remolacha o acelga, sin embargo la producción termina con la cosecha temprana de las plantas, o sea, cuando las hojas todavía no presentan el limbo foliar completamente expandido. En Brasil no hay normas oficiales de clasificación para definir el momento de cosecha para plantas tipo baby leaf, lo que hace que el producto se comercialice en diferentes tamaños dependiendo de la especie y del tipo de uso (hojas sueltas o planta entera). Es posible la comercialización de hojas entre 5 y $15 \mathrm{~cm}$ de longitud. En otros países, la cosecha es determinada cuando la longitud de la hoja está entre 5 y $9 \mathrm{~cm}$ desde el peciolo hasta la punta del limbo foliar (Martínez-Sánchez et al., 2012)

La perspectiva de aumento del consumo de baby leaf en Brasil es grande. En la actualidad, parte de lo que se comercializa es producido por el cultivo hidropónico (Purquerio e Melo, 2011) y parte se produce utilizando bandejas de poliestireno en invernaderos.

En Curitiba - PR (Brasil) y regiones cercanas, los supermercados están ofreciendo una mezcla de miniplantas (remolacha, berro, lechuga rizada, acelga) en una sola bolsa de plástico, similar a la utilizada para la comercialización de los productos hidropónicos. Las plántulas presentan 6 a 8 hojas jóvenes, con el sistema radicular envuelto por sustrato, por haber se desarrollado en bandejas de poliestireno. Sin embargo, esa manera de cultivo tiene un precio más alto por producto comparado al cultivo directamente en el suelo, como es realizado en otros países (Martínez-Sánchez et al., 2012), que incluso utilizan el proceso de siembra y cosecha mecanizados. En la actualidad, los estudios sobre el tema se centran más en los aspectos de la pos-cosecha (Nicola et al., 2006), especialmente la calidad fitosanitaria del producto. Sin embargo, en Brasil no hay estudios evaluando la siembra directa en el suelo, tampoco utilizando diferentes sistemas de protección de las plantas.

La utilización de cultivo protegido con túnel bajo cubiertos con polietileno de baja densidad (PEBD) o con agrotextil puede ser una alternativa al cultivo al aire libre para las regiones con condiciones climáticas adversas para la producción de baby leaf. Además del uso de ambientes protegidos, otro factor que interfiere con el desarrollo de plantas y posibilita la optimización de la producción es el marco de plantación utilizado, lo que permite el control del número de plantas por área de cultivo. Poco se sabe sobre técnicas adecuadas para la producción de baby leaf en las diferentes condiciones climáticas de Brasil, especialmente para el cultivo del berro de tierra (Barbarea verna).

Con base enlo anterior, el trabajo tuvo como objetivo evaluar los sistemas de cultivo y marcos de plantación para la producción de baby leaf de berros de tierra en Ponta Grossa-PR, sur de Brasil.

\section{Materiales Y Métodos}

El experimento se realizó en Ponta Grossa- PR, Brasil $\left(25^{\circ} 05^{\prime} 37\right.$ "S, 5003'35"O y $850 \mathrm{~m}$ de altitud). La región presenta promedios anuales de $1422 \mathrm{~mm}$ de precipitación y unos $75 \%$ de humedad relativa del aire (Godoy et al., 1976). El cultivo se estableció en un suelo tipo Cambisolo distrófico de textura arcillosa. El análisis químico del suelo antes del experimento presentó $\mathrm{pH}$ de $5,2\left(\mathrm{CaCl}_{2}\right), 30 \mathrm{~g} \mathrm{dm}^{-3}$ de carbono orgánico, 50,8 $\mathrm{mg} \mathrm{dm}^{-3}$ de fósforo (Mehlich 1), 0,8; 5,9 y 2,3 $\mathrm{cmol}_{\mathrm{c}} \mathrm{dm}^{-3}$, respectivamente, de K, Ca y $\mathrm{Mg}$ y $59,6 \%$ de saturación de bases. No se realizó aporte químico en el suelo debido a las características adecuadas para el cultivo (plantas pequeñas y de ciclo corto).

La siembra del berro de tierra se produjo en el 26 de octubre (primavera) directamente en el suelo. El diseño experimental fue bloques al azar con tratamientos dispuestos en parcelas sub-divididas con cinco repeticiones. Los tratamientos consistieron en la combinación de tres ambientes de cultivo y tres marcos de plantación.

Los ambientes de cultivos fueron Túnel bajo cubierto con agrotextil- TA (polipropileno blanco con $17 \mathrm{~g} \mathrm{~m}^{-2}$ de gramatura); Túnel bajo cubierto con plástico - TP (polietileno de baja densidad - 100 micras de espesor y con tratamiento anti-UV) y cultivo en Aire Libre (AL). Los marcos de plantación (entre plantas X entre hileras) fueron $15 \mathrm{~cm} \mathrm{x} 10 \mathrm{~cm}$ (E1); 15 $\mathrm{cm} \quad$ x $7 \mathrm{~cm}$ (E2) y $10 \mathrm{~cm}$ x $7 \mathrm{~cm}$ (E3), que 
corresponden a las densidades de 66,7; 95,2 y 142,9 plantas $\mathrm{m}^{-2}$, respectivamente.

Los marcos de plantación y las correspondientes densidades de plantas se definieron utilizando la referencia de los menores valores de recomendación para plantío convencional del berro de tierra (33 plantas $\mathrm{m}^{-2}$ ). Así, como las plantas de baby leaf presentan tamaño reducido en relación a las convencionales, se optó por aumentar, aproximadamente, las densidades de siembra en cerca de $2 ; 3$ y 4 veces $(67,95$ y 142 plantas $\mathrm{m}^{-2}$, respectivamente), con diferentes diseños de marcos de plantación.

El microclima en cada ambiente de cultivo fue caracterizado mediante el registro continuo de la radiación fotosintéticamente activa - PAR (Quantum Sensor LI190SB LiCor) y de la temperatura del aire (20 $\mathrm{cm}$ por encima del suelo) utilizándose termistores (modelo 107 - Campbell Sci).Los sensores se conectaron a un sistema de adquisición de datos (CR23X, Campbell Sci.), programado para tomar datos a cada minuto, almacenando la media a cada hora. Los datos de la radiación PAR se registraron en la unidad de

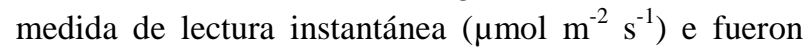
integralizados para mol $\mathrm{m}^{-2} \mathrm{día}^{-1}$. Después los datos fueron transformados en $\mathrm{MJ} \mathrm{\textrm {m } ^ { - 2 }}$ día $^{-1}$, según lo propuesto por Thimijan y Heins (1983) por la ecuación: $\mathrm{PAR}=\Sigma$ diário $\left[\mathrm{PAR}\left(\mu \mathrm{mol} \mathrm{m} \mathrm{s}^{-2}\right) \mathrm{t}(\mathrm{s}) 4,57^{-1}\right] 10^{-6}(\mathrm{MJ}$ $\mathrm{m}^{-2}$ día $\left.{ }^{-1}\right)$, donde 4,57 es el valor de conversión (Beckmann et al., 2006).

Se necesario, se llevó a cabo la irrigación por aspersión, manteniéndose las coberturas de los túneles abiertas para la entrada del agua. El control de malezas se realizó manualmente. La cosecha se realizó a los 42 días después de la siembra (DDS), cuando las plantas presentaban unas medias de 05 hojas y altura $\geq 10 \mathrm{~cm}$ (longitud media para comercialización de baby leaf en Brasil), siendo este el momento de cosecha que se consideró para baby leaf de berros de tierra.
Fueron cosechadas 08 plantas por parcela útil para la evaluación de la altura de planta (AP), número de hojas por planta (NHP), índice de área foliar (IAF), biomasas fresca (BF) y seca (BS) de plantas y el rendimiento total (RT) de cultivo. Los datos fueron evaluados realizando un Análisis de Varianza y las medias entre tratamientos comparadas mediante test de Tukey al 5\% de probabilidad, utilizando se el software SISVAR - UFLA 4,6 (Ferreira, 2011).

\section{Resultados Y Discusión}

Las interacciones entre ambientes de cultivo y marco de plantación no fueron significativas para las características evaluadas resultando en la independencia entre los factores estudiados. Por lo tanto, cuando se analizaron por separado, hubo significancia para AP, NHP, IAF, BF, BSy RT entre los ambientes de cultivo (Tabla 1) y para NHP, IAF, BF yRT entre los marcos de plantación (Tabla 2).

Los ambientes de cultivo con túnel plástico (TP) y con túnel de agrotêxtil (TA) proporcionaron mayor altura de plantas (AP) en relación al cultivo al aire libre (Tabla 1), con incrementos de 40,5 y 32,5\% para los cultivos bajo TA y TP, respectivamente. Además, las plantas cultivadas en AL resultaron en valores más bajos de NHP en comparación con los otros ambientes de cultivo (Tabla 1). Es decir, el mayor crecimiento de las plantas protegidas (TP y TA) tipo baby leaf fue seguido por un mayor desarrollo de la misma, ya que hubo variación del NHP entre las plantas en diferentes ambientes de cultivo. En igual sentido, Sá e Reghin (2008) estudiaron el crecimiento y rendimiento de la escarola (ciclo completo de la planta) cultivadas bajo túnel plástico, bajo agrotextil (cubierta flotante) y en aire libre y plantearon un mayor crecimiento de las plantas cultivadas bajo el túnel.

Tabla 1. Altura de la planta (AP), número de hojas (NHP), índice de área foliar (IAF), masas fresca (MF) y seca de la planta (MS) y rendimiento total (RT) de Baby leaf de berro de tierra cultivado en diferentes ambientes de cultivo.

\begin{tabular}{lcccccc}
\hline Ambientes & AP (cm) & NHP & IAF & $\begin{array}{c}\text { MF } \\
\left(\text { planta }^{-1}\right)\end{array}$ & $\begin{array}{c}\text { MS } \\
\left(\mathrm{g} \mathrm{planta}^{-1}\right)\end{array}$ & $\begin{array}{c}\text { RT } \\
\left(\mathrm{g} \mathrm{m}^{-2}\right)\end{array}$ \\
\hline Aire Libre (AL) & $12,6 \mathrm{~b}^{*}$ & $9,0 \mathrm{~b}$ & $1,1 \mathrm{~b}$ & $3,5 \mathrm{~b}$ & $0,50 \mathrm{~b}$ & $349 \mathrm{~b}$ \\
Túnel Plástico (TP) & $16,7 \mathrm{a}$ & $10,0 \mathrm{a}$ & $1,6 \mathrm{a}$ & $5,2 \mathrm{a}$ & $0,62 \mathrm{a}$ & $524 \mathrm{a}$ \\
Túnel Agrotextil (TA) & $17,7 \mathrm{a}$ & $10,0 \mathrm{a}$ & $1,7 \mathrm{a}$ & $5,4 \mathrm{a}$ & $0,70 \mathrm{a}$ & $520 \mathrm{a}$ \\
\hline CV $(\%)$ & 6 & 3 & 7 & 10 & 5 &
\end{tabular}

* Medias seguidas de la misma letra en la columna no difieren por la prueba de Tukey al 5\% de probabilidad. CV = coeficiente de variación.

Tabla 2. Altura de planta (AP), número de hojas por planta (NHP), índice de área foliar (IAF), biomasa fresca (BF) y biomasa seca (BS) y el rendimiento total (RT) de baby leaf de berro de la tierra respecto a diferentes marcos de plantación.

\begin{tabular}{lcccccc}
\hline $\begin{array}{l}\text { Marco de Plantación } \\
(\mathrm{cm} \mathrm{x} \mathrm{cm})\end{array}$ & $\begin{array}{c}\text { AP } \\
(\mathrm{cm})\end{array}$ & NHP & IAF & $\begin{array}{c}\text { BF } \\
\left(\mathrm{g} \mathrm{planta}^{-1}\right)\end{array}$ & $\begin{array}{c}\text { BS } \\
\left(\mathrm{g} \mathrm{planta}^{-1}\right)\end{array}$ & $\begin{array}{c}\text { RT } \\
\left(\mathrm{g} \mathrm{m}^{-2}\right)\end{array}$ \\
\hline E1 $(15 \times 10)$ & $15,4 \mathrm{a}^{*}$ & $10,2 \mathrm{a}$ & $1,1 \mathrm{c}$ & $5,3 \mathrm{a}$ & $0,67 \mathrm{a}$ & $347 \mathrm{c}$ \\
E2 $(15 \times 7)$ & $15,8 \mathrm{a}$ & $9,3 \mathrm{~b}$ & $1,5 \mathrm{~b}$ & $4,6 \mathrm{ab}$ & $0,61 \mathrm{a}$ & $473 \mathrm{~b}$ \\
E3 $(10 \times 7)$ & $15,9 \mathrm{a}$ & $9,4 \mathrm{ab}$ & $1,9 \mathrm{a}$ & $4,2 \mathrm{~b}$ & $0,55 \mathrm{a}$ & $598 \mathrm{a}$ \\
\hline
\end{tabular}




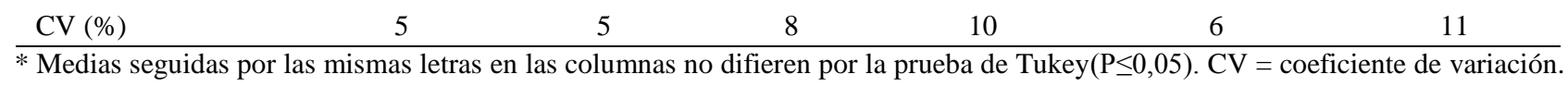

El mayor crecimiento de plantas de baby leaf en un ambiente protegido fue acompañado por valores más altos debio masa fresca (BF) y del índice de área foliar (IAF) en comparación con plantas cultivadas en aire libre (Tabla 1). Una tendencia similar se produjo para la biomasa seca de plantas (BS) bajo cultivo en AG y TP (Tabla 1) que también presentaran valores más altos que los cultivos en AL. Estos datos indican que los materiales utilizados en los sistemas de protección de las plantas no han reducido la radiación fotosintéticamente activa (PAR) incidente a niveles dañosos al desarrollo del cultivo (Figura 1). No hay indicación de etiolación de las plantas como respuesta a la reducción de la radiación PAR incidente por el uso de la protección.

Los valores más bajos de PAR bajo los ambientes protegidos frente a $\mathrm{AN}$ tienen relación con la transmisividad de los materiales de protección, que era 84 y $91 \%$ para AT y AG, respectivamente. Según la FAO (1990), el límite de la radiación total incidente debajo de la cual la mayoría de las plantas no producen el mínimo de foto asimilados necesarios para su mantenimiento (definido como límite trófico) es 8,4 MJ $\mathrm{m}^{-2}$ día $^{-1}$. Según la relación determinada por Assis e Mendez (1989) para ambiente natural, la radiación PAR corresponde a $47 \%$ de ese valor $\left(3,9 \mathrm{MJ} \mathrm{m}^{-2}\right.$ día $\left.^{-1}\right)$. Se encontró que en los tratamientos donde se utilizaron los sistemas de protección, los valores medios de PAR incidente se quedaron mayores que aquellos recomendado por la FAO (1990), es decir, 4,6 y 5,0 MJ $\mathrm{m}^{-2}$ día $^{-1}$, respectivamente, para TA y TP (Figura 1).

El mayor crecimiento de las plantas cultivadas en ambiente protegido está relacionado a la mejor utilización de la radiación solar y del balance de energía proporcionado por la protección, resultando en cambios de la temperatura del aire. A lo largo del ciclo de cultivo hubo valores medios de temperatura del aire de 18, $19 \mathrm{y}$ $17{ }^{\circ} \mathrm{C}$, respectivamente, para el TA, TP y AN (Figura 2). La media diaria de la temperatura del aire bajo TP llegó a más de $3{ }^{\circ} \mathrm{C}$ en los días más calurosos (entre 27 y 32 DAS) en comparación con AN.

Aunque en AN fue verificado mayores valores de radiación PAR incidente (Figura 1), los valores más bajos de temperatura del aire (Figura 2) pueden haber contribuido para la actividad metabólica más lenta de estas plantas en comparación con aquellas cultivadas bajo ambientes protegidos. Los resultados son plantas más pequeñas para todas las características discutidas anteriormente (Tabla 1). Las modificaciones microclimáticas resultantes del uso de cada sistema de protección respecto $\mathrm{AL}$ interfieren directamente en el metabolismo de las plantas, lo que permite una variación en el ciclo de cultivo. Así, la opción por el uso de sistemas cultivo protegidos sería una alternativa para la cosecha de plantas con mayor precocidad.

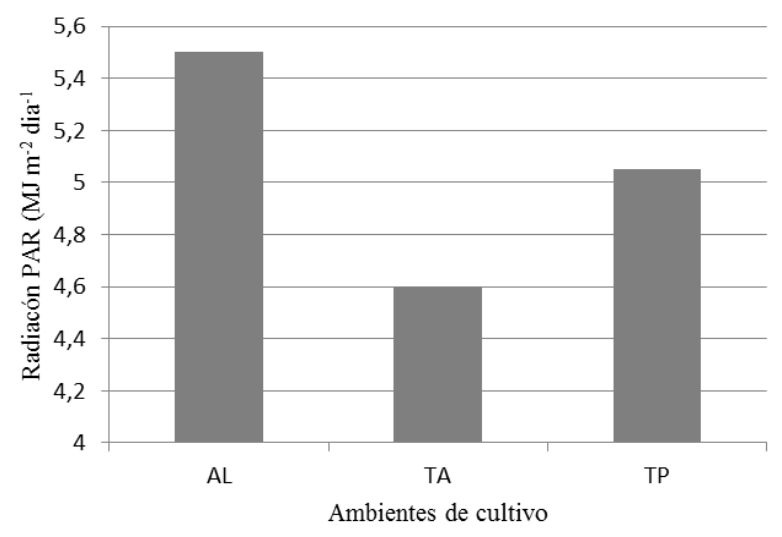

Figura 1. Por medio de la radiación fotosintéticamente activa (PAR) incidente a lo largo del ciclo de baby leaf de berro de tierra cultivado al aire libre (AL), bajo túnel de agrotextil (TA) y bajo túnel plástico (TP). (Photosynthetically active radiation (PAR) incident along the cycle of land cress baby leaf growing in natural conditions (AL), nonwoven tunnel (TA) and plastic tunnel (TP)).

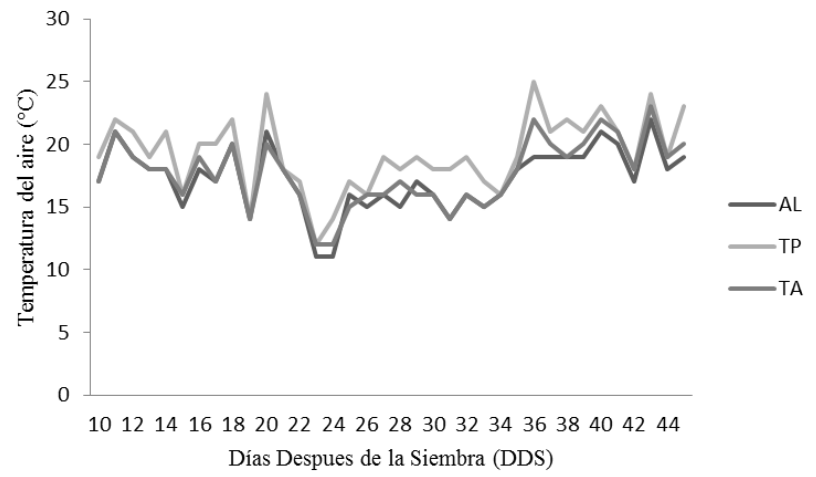

Figura 2. Temperatura media del aire a lo largo del ciclo de Baby leaf de berro de la tierra cultivado bajo Túnel Plástico (TP), Túnel Agrotextil (TA) y en Aire Libre (AL).(Air temperature along the cycle of land cress baby leaf growing in natural conditions (AL), nonwoven tunnel (TA) and plastic tunnel (TP)).

Como resultado también se encontraron niveles más altos de rendimiento total (RT) para las plantas cultivadas en ambientes con protección (TP y TA) en comparación con los cultivados en AL. Losrendimiento promedio aumentaran $50 \%$ para las plantas cultivadas bajo TP y TA con respecto al cultivo en AL (Tabla 1). Resultados similares fueron observados por Pereira et al. (2003) que, estudiando el cultivo de rúcola (ciclo completo de la planta) bajo TP e AL, encontraron que la producción fue un $50 \%$ superior en TP en comparación 
con AL. Del mismo modo, Barros Junior et al. (2004), en experimentos con lechuga (ciclo de cultivo completo) cultivada bajo TA, encontraron mayor rendimiento total en comparado al cultivo en ambiente natural. Parte de estos resultados, los autores relacionaron con la mejor utilización de la radiación solar incidente y el incremento de la temperatura del aire bajo ambiente protegido.

Respecto a los marcos de plantación fueron verificadas diferencias entre las características NHP,IAF, BF y RT de las plantas (Tabla 2).

La altura de planta entre los marcos de plantación evaluados fue similar, sin embargo, las plantas cultivadas en E1 presentaron una ligera diferencia en el desarrollo (>NHP en comparación con E2), pero con hojas más pequeñas ( $<\mathrm{IAF})$, aún que resultaron en mayores valores de $\mathrm{BF}$ de plantas individuales (Tabla 2). Por otro lado, este resultado no justifica la elección del marco de plantación E1 como el más adecuado para la producción de baby leaf de berro porque la opción de mayor densidad de plantas (E3) resultó en un mayor rendimiento total (RT) en comparación con otros marcos de plantación (Tabla 2). Además, los mayores valores de IAF encontrados para el cultivo en mayor densidad (E3) resultaron en hojas más tiernas con calidad comercial.

\section{Conclusión}

La producción de baby leaf de berros de tierra fue viable para el cultivo bajo túneles que utilizaron plástico o agrotextil como material de protección durante la primavera de Ponta Grossa - PR, utilizando marco de plantación $10 \times 7 \mathrm{~cm}$ (E3) entre plantas. Estas técnicas de cultivo resultaron en aumento del rendimiento total y de la calidad de las plantas comerciales.

\section{Literatura Citada}

Assis, F.N., Mendez, M.E.G., 1989. Relação entre radiação fotossinteticamente ativa e radiação global. Pesquisa Agropecuária Brasileira, 2, 797-800.

Barros Junior, A.P., Grangeiro, L.C., Bezerra Neto, F., Negreiros, M.Z., Souza, J.O., Azevedo, P.E., Medeiros, D.C., 2014. Cultivo da alface em túneis baixos de agrotêxtil. Horticultura Brasileira, 22, 801-803.

Beckmann, M.Z., Duarte, G.R.B., Paula, V.A., Mendez, M.E.G. De., Peil R.M.N., 2006. Radiação solar em ambiente protegido cultivado com tomateiro nas estações verão-outono no Rio Grande do Sul. Ciência Rural, 36, 86-92.

Food and Agriculture Organization of the United Nations. Protected cultivation in the Mediterranean climate. Rome: FAO, 1990.313 p.(Plant Production and Protection Paper, 90).

Ferreira, D.F., 2011. Sisvar: a computer statistical analysis system. Ciência e Agrotecnologia, 35, 1039-1042.

Godoy, H., Correa, A.R., Santos, D., 1976. Clima do Paraná, in: IAPAR. Manual agropecuário para o Paraná. IAPAR, Londrina, pp.17-36.

Martínez-Sánchez, A., Luna, M.C., Selma, M.V., Tudela, J.A., Abad, J., Gil, M.I., 2012. Baby-leaf and multi-leaf of green and red lettuces are suitable raw materials for the fresh-cut industry. Postharvest Biology and Technology, 63, 1-10.

Nicola, S., Fontana, E., Torassa, C., Hoeberechts, J., 2006. Fresh-cut produce: postharvest critical issues. Acta Horticuturae, 712, 223-230.

Pereira, E.R., Silva, I.J.O., Moura, D.J., 2003. Alterações microclimáticas em túneis baixos com plástico perfurado no cultivo da rúcula em época de verão e de outono. Engenharia Agrícola, 23, 407-414.

Purquerio, L.F.V., Melo, P.C.T., 2011. Hortaliças Pequenas e saborosas. Horticultura Brasileira, 29, 1-1.

Thimijan, R.W., Heins, R.D., 1983, Photometric, radiometric, and quantum light units of measure: a review of procedures for interconversion. HortScience, 18, 818-822.

Sá, G.D., Reghin, M.Y., 2008, Desempenho de duas cultivares de chicória em três ambientes de cultivo. Ciência e Agrotecnologia,32,378-384. 